“C 2016 IEEE. Personal use of this material is permitted. Permission from IEEE must be obtained for all other uses, in any current or future media, including reprinting/republishing this material for advertising or promotional purposes, creating new collective works, for resale or redistribution to servers or lists, or reuse of any copyrighted component of this work in other works." 


\title{
Angled Sensor Configuration Capable of Measuring Tri-Axial Forces for pHRI
}

\author{
Christian Reeks, Marc G. Carmichael, Dikai Liu and Kenneth J. Waldron
}

\begin{abstract}
This paper presents a new configuration for single axis tactile sensor arrays molded in rubber to enable tri-axial force measurement. The configuration requires the sensing axis of each sensor in the array to be rotated out of alignment with respect to external forces. This angled sensor array measures shear forces along axes in a way that is different to a planar sensor array. Three sensors using the angled configuration $\left(22.5^{\circ}, 45^{\circ}\right.$ and $\left.67.5^{\circ}\right)$ and a fourth sensor using the planar configuration $\left(0^{\circ}\right)$ have been fabricated for experimental comparison. Artificial neural networks were trained to interpret the external force applied along each axis $(\mathrm{X}, \mathrm{Y}$ and $Z$ ) from raw pressure sensor values. The results show that the angled sensor configuration is capable of measuring tri-axial external forces with a root mean squared error of $1.79 \mathrm{~N}$, less error in comparison to the equivalent sensor utilizing the planar configuration $(4.52 \mathrm{~N})$. The sensors are then implemented to control a robotic arm. Preliminary findings show angled sensor arrays to be a viable alternative to planar sensor arrays for shear force measurement; this has wide applications in physical Human Robot Interaction (pHRI).
\end{abstract}

\section{INTRODUCTION}

In the past, industrial robots were too dangerous to operate near humans. Initially physical barriers were placed between the robot and user. Soon after, robots were able to detect when a human entered their workspace. This was achieved most commonly with cameras and light screens [1]. Now, with the rise of pHRI, robots need a way of detecting the users' intent. Tactile sensors are suited for intent detection because the sense of touch is an intuitive expression of intention. A force-torque $(\mathrm{F} / \mathrm{T})$ sensor mounted on the robot is one method to measure forces from physical interaction. The drawback of this method is that each point of contact requires its own F/T sensor. A sensor skin or array of sensors that cover the robot would be a practical solution for this problem.

A sensor skin refers to a continuous sensor or a matrix of modular sensors. Single axis sensor skins have been proposed and achieved through a variety of methods. Conductive and piezoelectric materials arranged in a lattice structure allow for the location of the normal force to be estimated [2], [3]. Optical sensors covered in polyurethane rubber (PUR) allow for quick and wire free installation of a sensor skin [4]. Sensor skins capable of measuring shear forces are currently available but most have a very limited sensing range in shear directions [5]. CellulARSkin uses individual sensing units that detect not only normal force but also temperature, acceleration and proximity [6], [7]. The limitation for most sensor skins is their inability to sense

The authors are with the Centre for Autonomous Systems (CAS), Faculty of Engineering and IT, University of Technology Sydney (UTS), NSW 2007, Australia, corresponding email: Christian.Reeks@uts.edu.au shear forces. The ability to measure shear force opens up the possibility of implementing new modalities for human-robot interaction.

Existing research has developed three axis force sensors using a diverse range of methods. The most common methods involve force channeling structures with specific geometries or material properties placed over a flat array of sensing elements [8]-[18]. External forces are applied to the structure which channels the forces in to the sensing element. Wang and Beebe apply forces to a cube-like structure that deforms a diaphragm underneath. Four piezoresistors detect the stresses in the diaphragm and the tri-axial forces are resolved [8]. Multiple capacitors embedded in polydimethylsiloxane (PDMS) have been shown to detect tri-axial forces of up to 2 newtons [9]-[12]. Optoelectronics have been used to create a deformable force sensor. A silicone dome is used for the tactile interface. The dome has small cavities that sit over the top of a PCB. Inside each of the cavities is a LED and phototransistor. During an interaction the dome deforms along with the cavities inside. This causes a varying light intensity to be detected by the phototransistor and the external force can be resolved [13] [15]. Liquid metal piezoresistors encased in PDMS have been shown to measure shear forces [16], [17]. A table shaped contact plate with each of the 4 legs located on top of a strain gauge has been used to obtain tri-axial force measurements [18]. To the knowledge of the author there has not been a sensor developed using the proposed angled sensor configuration.

The single axis tactile sensor used in this paper is a 'Takktile' barometric pressure sensor encased in PUR [19]. A tactile sensor array on a flexible skin was made using these pressure sensors. However, the skin was only designed to measure normal forces [20]. A sensor able to measure normal and shear forces was successfully fabricated and integrated into the footpad of a cheetah robot. The footpad sensor consists of a flat $3 \times 3$ array of Takktile sensors. During ground locomotion the footpad sensor deforms causing varied pressure values for the Takktile sensors in the array [21]. This is an example of a planar sensor array and allows the applied normal and shear forces to be predicted. In this work we present an alternative method to the planar sensor array for measuring shear forces with multiple single axis sensors.

The tactile element (taxel) developed in this paper uses a novel configuration of single axis sensors to measure normal and shear forces. The configuration can be applied in a modular fashion to create a sensor skin capable of measuring tri-axial forces. A deformable normal and shear measuring sensor skin that covers a robot would be well suited for pHRI. 


\section{SENSOR DESIGN}

\section{A. Planar Sensor Configuration verses Angled Sensor Configuration}

Single axis sensors cannot simultaneously measure normal and shear forces. An array of single axis sensors can exploit the edge effect caused by the deformation of the PUR. When a shear force is applied to a material, tensile and compressive forces are produced away from and in front of the shear direction respectively. In elastic materials such as PUR these tensile and compressive forces manifest themselves at the edge of the material. Pressure sensors located at the edge detect tensile force as a negative pressure while compressive force is detected as a positive pressure (Fig. 1). Measurement of this edge deformation allows the shear loads applied to be estimated. This method in practice has shown a RMSE of less than 5 newtons in shear [21].

The sensor configuration proposed in this paper is used to create an angled sensor array. The angled configuration assumes that all forces applied will be with respect to (w.r.t) the world coordinate frame (Fig. 2). When the sensor coordinate frame is aligned with the world coordinate frame and a single axis sensor is in the $\mathrm{X}-\mathrm{Y}$ plane, the sensor can only detect forces along the Z-Axis. Rotating the sensor about the $\mathrm{X}$ - or $\mathrm{Y}$-Axes causes the sensor frame Z-Axis to diverge from the world frame Z-Axis. Any force in the world frame can be decomposed w.r.t. the sensor frame. The decomposed world frame force will have components along each of the sensor frame axes. One of these component forces will be normal to the sensor, even if the initial force was purely shear.

\section{B. Sensor Composition}

Three angled sensor taxels and a planar sensor taxel were fabricated for testing. Each taxel consisted of a 3D printed Acrylonitrile Butadiene Styrene (ABS) plastic base, four barometric pressure sensors and a PUR top lay of Shore hardness 20A (Fig. 3). The four barometric pressure sensors were laid out in a Maltese cross format. The sensor openings were always located closer to the interaction surface of the taxel. The amount of rubber between the interaction surface and the sensor opening was kept a constant $6 \mathrm{~mm}$ for each taxel. . The angled configurations chosen for testing were $0^{\circ}$, $22.5^{\circ}, 45^{\circ}$ and $67.5^{\circ}$. The $0^{\circ}$ taxel serves as a reference or control for testing. This taxel has no tilt and relies on edge deformation for measuring shear forces. Each taxel was made with commercially available materials and the only specialized tool required for assembly is a vacuum chamber. Each sensor has a base size of $31 \times 31 \mathrm{~mm}$ to make assembling and testing of the sensors more convenient. The $0^{\circ}, 22.5^{\circ}, 45^{\circ}$ and $67.5^{\circ}$ taxels have heights of $15,17,19$ and $24 \mathrm{~mm}$ respectively.

\section{SENSOR CHARACTERIZATION}

The taxels must be characterized and calibrated before meaningful force values can be obtained. Forces of known direction and magnitude were applied to each taxel. The pressure signals from the taxel and the applied forces were recorded. To gather the true force values a six axis F/T
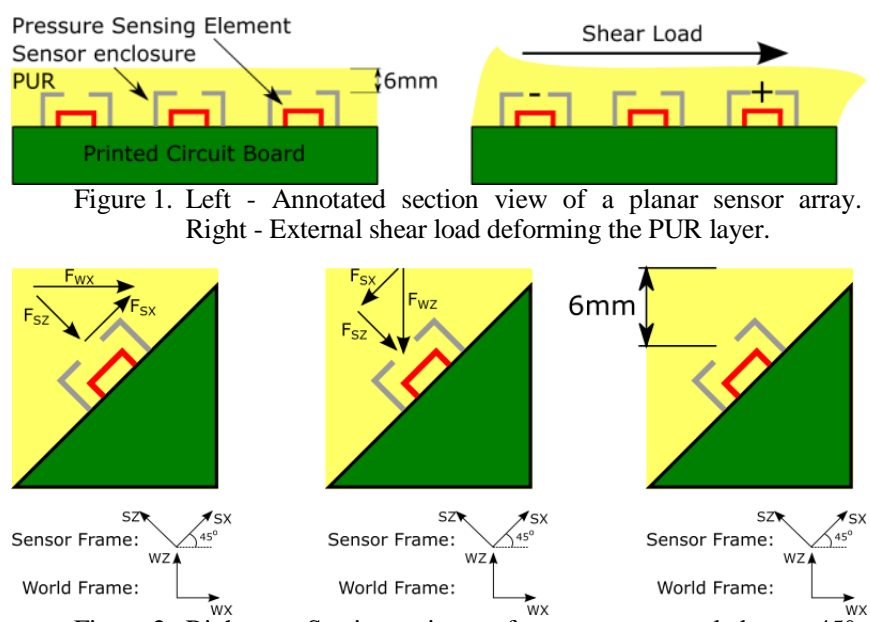

Figure 2. Right - Section view of a sensor angled at $45^{\circ}$. Center - External normal force decomposed into component forces w.r.t. the sensor frame. Left - External shear force being decomposed into sensor frame forces.

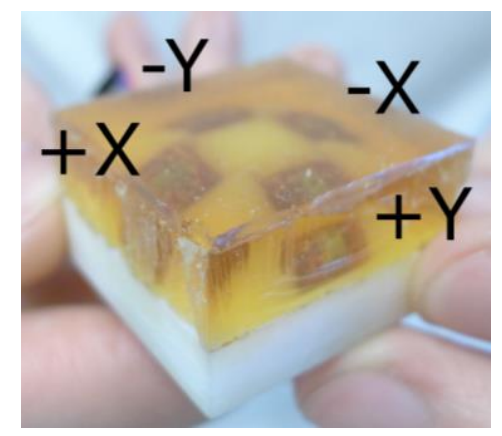

Figure 3. $45^{\circ}$ taxel with 3D printed base and cast in PUR. Each single axis sensor is labelled according to the axis it belongs to.

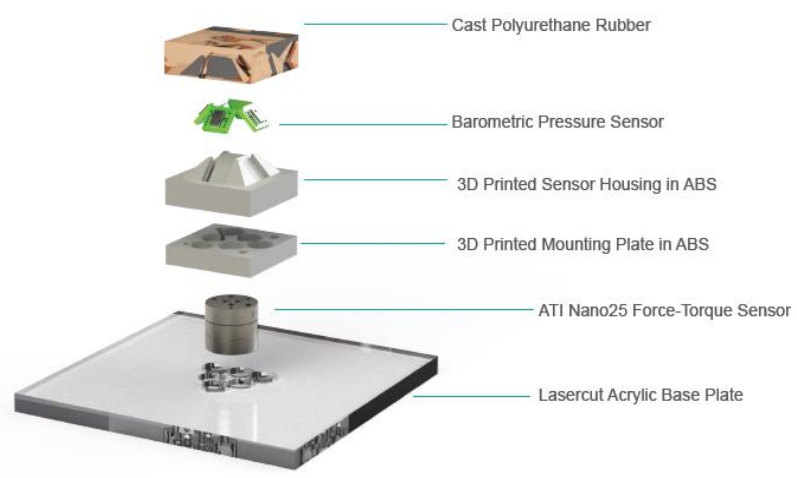

Exploded View

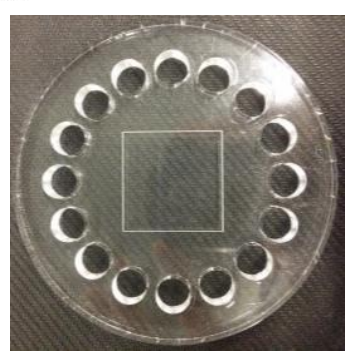

Figure 4. Top - Annotated exploded view of the experimental set up. Bottom - Perspex plate used for applying shear loads. The etched square is aligned with the top face of the taxel. 
sensor (ATI Industrial Automation Nano 25) was mounted to the taxel. To generate forces consistent with human-robot interaction, a human subject applied external forces by hand to the sensor. The palm of the hand would be a likely way to exert forces onto the taxel. We assume that the palm is roughly planar. This determines that all forces applied will be through a planar object. A Perspex plate was laser cut to fulfill this purpose (Fig. 4(Bottom)). The plate sits on top of the cast rubber and friction is used to transmit shear loads to the taxel. Holes are located around the circumference of the circle at $22.5^{\circ}$ increments to accommodate the hook of a spring gauge for applying shear forces.

The data recorded gives an indication of the sensing range for the taxels. During data collection shear loads of up to 40 newtons were applied without saturating or damaging the taxels. The raw data shows similar behavior between the planar and angled sensor arrays when measuring shear forces. Raw pressure values and X-Axis force from the F/T sensor for the $0^{\circ}$ planar sensor array and the $45^{\circ}$ angled sensor array are shown in Figures 5 and 6 respectively. The two high magnitude mirrored curves in each of the figures are from the two pressure sensors located along the X-Axis. A correlation between the pressure values and forces is clearly visible from the graphs. An algorithm or mapping is needed to estimate the applied axial forces from the pressure values.

\section{A. Neural Network}

An artificial neural network was chosen to map sensor pressure values to the normal and shear forces. Neural networks have been successfully used to map pressures from a planar sensor array to axial forces [21]. The same neural network type and topology was used for this research. A Levenberg-Marquardt neural network was trained for each taxel. The network consists of one input layer, one hidden layer with ten nodes and one output layer. Normal and shear forces can be interpreted in real time by a trained neural network.

Data sets containing four pressure inputs and three force outputs were needed to train the neural network. 100,000 data points were gathered for each taxel. This included 20,000 of only normal force, 20,000 of pure shear force in each axis and 40,000 combinations of normal and shear forces. Additional data points were recorded to validate the trained neural network.

\section{RESULTS AND DISCUSSION}

All angled taxels achieve RMSE values lower than the planar taxel. Table I shows the RMSE between predicted and actual force values for each taxel. The $0^{\circ}$ planar taxel has the highest error values. Among the angled taxels $45^{\circ}$ has the lowest error in both Y- and Z-Axis. The regression plots in Fig. 7 corroborate with the errors in table I. All angled taxels obtained larger $\mathrm{R}$ values than the planar taxel. An ideal $R$ value of 1 is achieved when the network output is equal to the actual force. The $45^{\circ}$ taxel again achieves the best result of the four sensors. This suggests that $45^{\circ}$ is the optimal angle but a larger cohort of angles need to be tested before a definitive conclusion can be made.
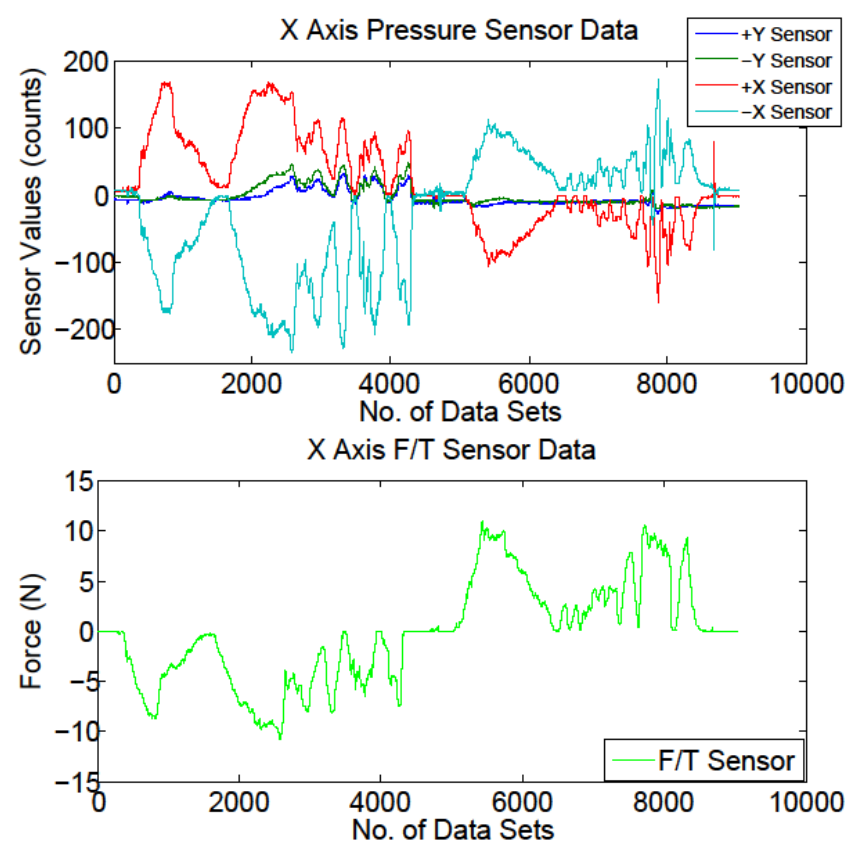

Figure 5. The top graph shows the X-Axis raw pressure values from the planar sensor array and the bottom graph shows the force readings from the $\mathrm{F} / \mathrm{T}$ sensor.

$\mathrm{X}$ Axis Pressure Sensor Data
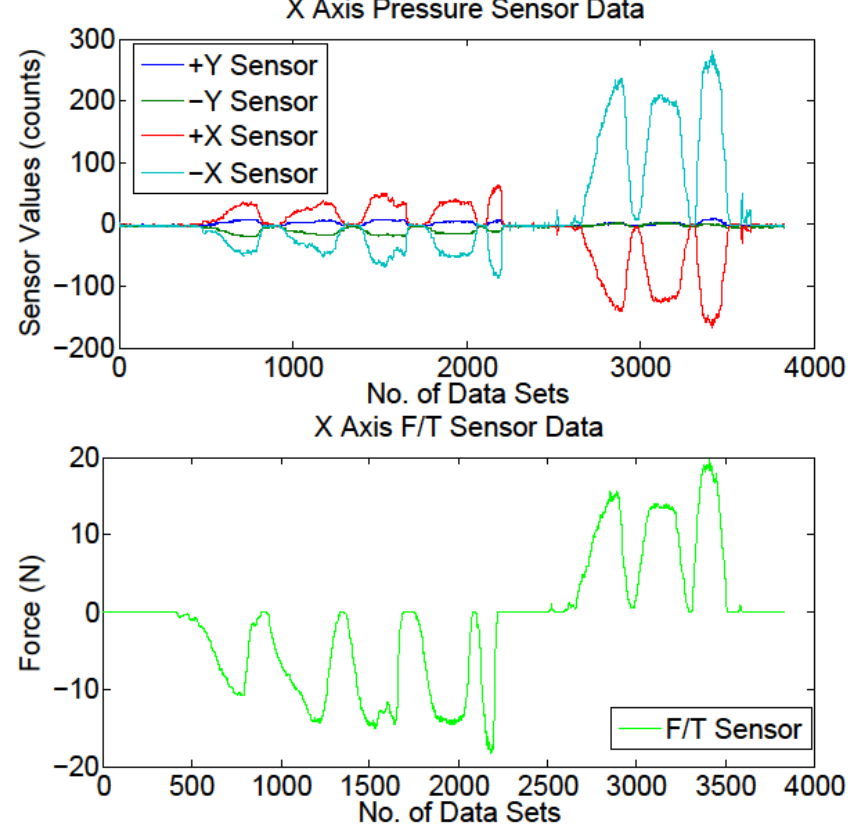

Figure 6. The top graph shows the $\mathrm{X}$ - Axis raw pressure values from the $45^{\circ}$ angled sensor array and the bottom graph shows the force readings from the F/T sensor.

Angled taxels have further advantages over the planar taxels. The footprint of an angled taxel will shrink as the angle gets larger. However the height of the taxel will also increase with the angle. The sensing range of angled taxels is larger than the range of planar taxels. This is due to the angled taxel only detecting a portion of an applied force as described in section II A. Specific applications may require sensitivities or ranges that differ from the values reported here. These values can be fine-tuned by varying the rubber hardness and thickness. 


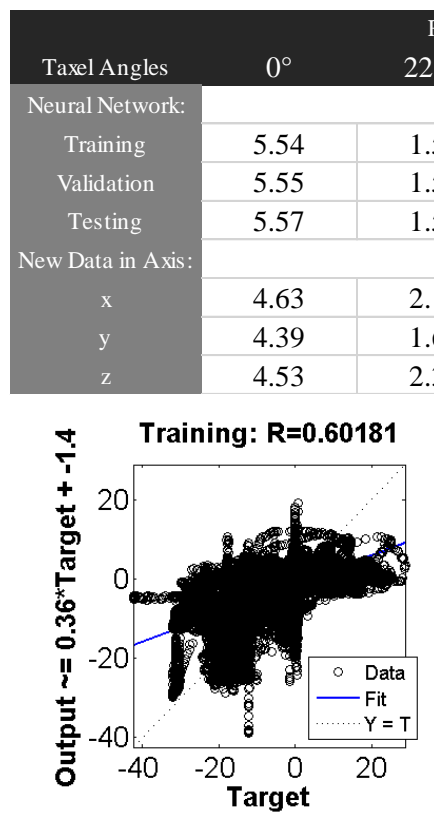

RMSE (Newtons)

$22.5^{\circ} \quad 45^{\circ}$

$67.5^{\circ}$

\begin{tabular}{l|l|r|}
1.55 & 1.22 & 1.21 \\
\hline 1.59 & 1.23 & 1.20
\end{tabular}

\begin{tabular}{l|l|l|}
1.59 & 1.23 & 1.20
\end{tabular}

\begin{tabular}{l|l|l|}
1.52 & 1.24 & 1.13 \\
\hline
\end{tabular}

2.12

2.13

1.34

\begin{tabular}{l|l|l|}
1.60 & 1.24 & 1.58 \\
\hline
\end{tabular}

\begin{tabular}{l|l|l|}
2.34 & 1.74 & 2.00 \\
\hline
\end{tabular}
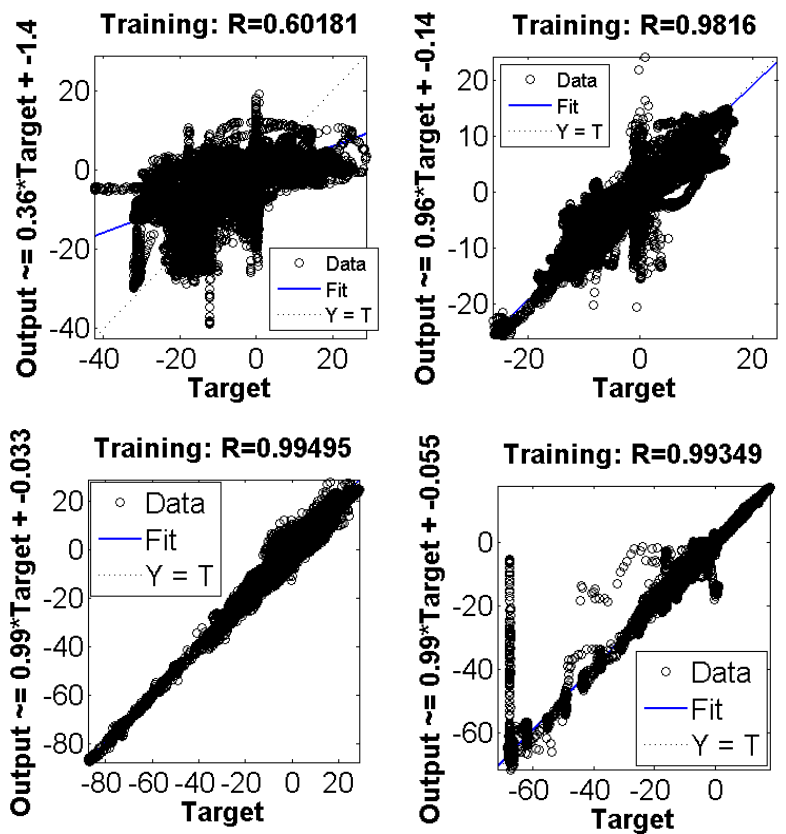

Figure 7. Neural Network training results. $0^{\circ}, 22.5^{\circ}, 45^{\circ}$ and $67.5^{\circ}$ from top left, top right, bottom left and bottom right respectively. Figures 8,9 and 10 will be in this format.

A point of interest is the difference in $\mathrm{X}$ - and Y-Axis error values. The design of a taxel is identical along the shear axes. It is expected that errors along these shear axes should also be identical. A possible cause of this discrepancy may be the barometric pressure sensors individual properties (e.g. sensitivity). Another possible cause is imperfect fabrication of the taxels. An air bubble trapped within a barometer or PUR not thoroughly mixed would change the properties of the taxel. In the future ultrasonic probing may be used as a quality check after manufacture.

\section{A. Forces in Z-Axis}

Correctly identifying forces along a single axis should be straight forward for sensors designed to measure normal forces. The planar taxel does not follow this logic. Instead the predicted force does not change when a normal force is applied, see Fig. 8(a). This is because the $0^{\circ}$ neural network is incorrectly predicting the forces applied to the taxel as shear instead of normal. The predicted and measured forces from the $45^{\circ}$ taxel closely match, as seen in Fig. 8(b). The regression plots in Fig. 8(c) show a distinct difference in $\mathrm{R}$ values between the planar and angled taxels. These experimental results show angled taxels having less error

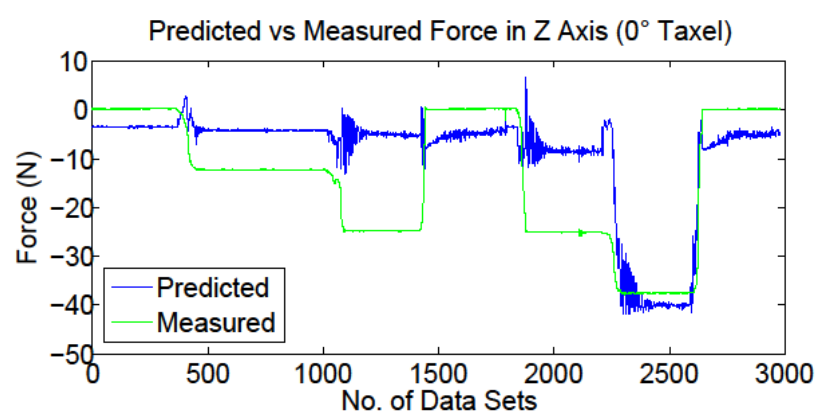

(a)

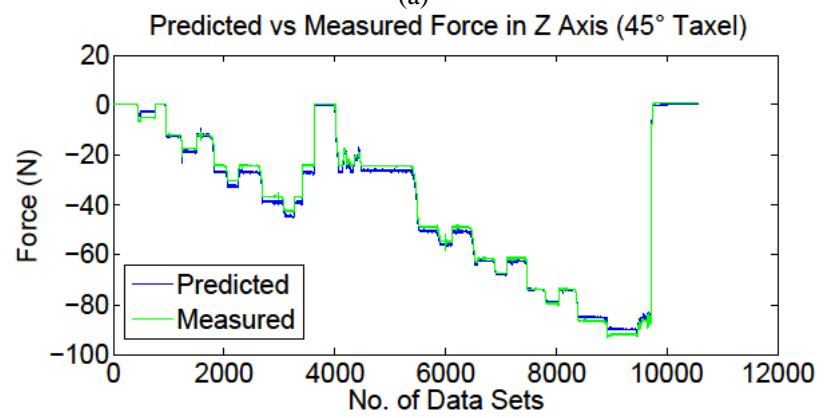

(b)
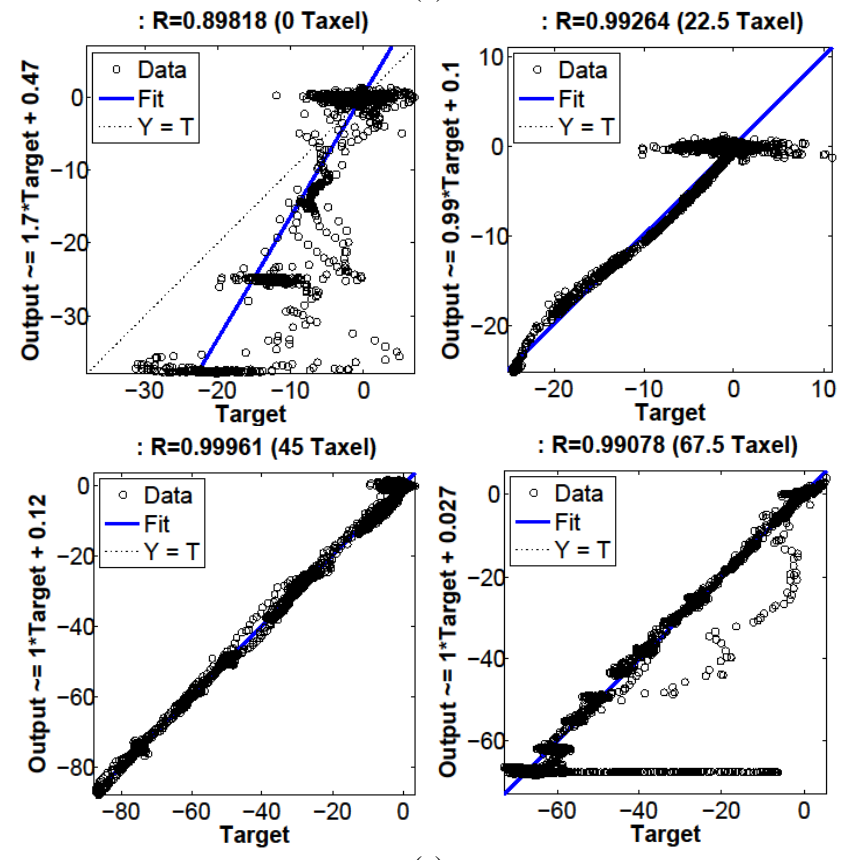

(c)

Figure 8. Results of testing Neural Networks with unseen data, normal direction only. (a) Predicted vs measured forces for the planar taxel. (b) Predicted vs measured forces for the $45^{\circ}$ angled taxel. (c) Regression plots.

and higher $\mathrm{R}$ values than the planar taxel when measuring forces along the Z-Axis.

\section{B. Forces in X-Axis}

Predicted force from both the $0^{\circ}$ planar and $45^{\circ}$ angled taxel express features similar to the measured force. The peaks in Fig. 9(a) and (b) suggest that both taxels correctly identify shear force direction along the X-Axis. The angled taxel in Fig. 9(b) shows less error in determining the 


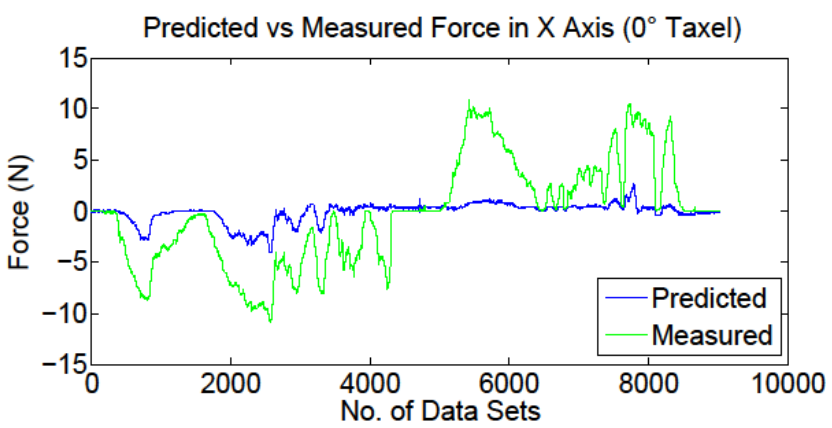

(a)

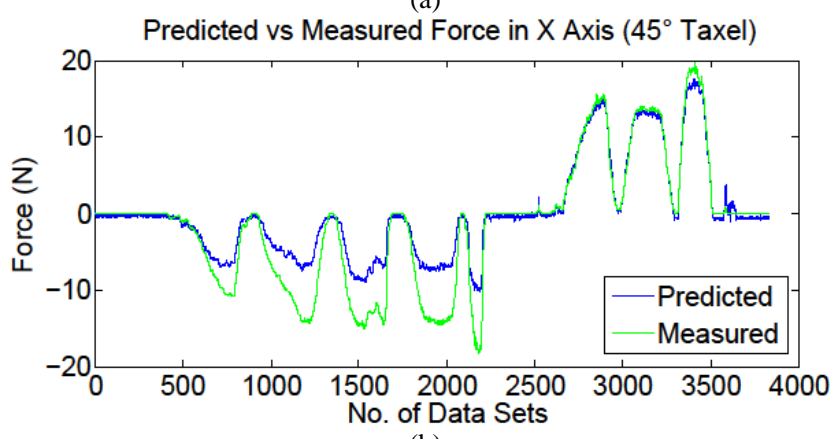

(b)
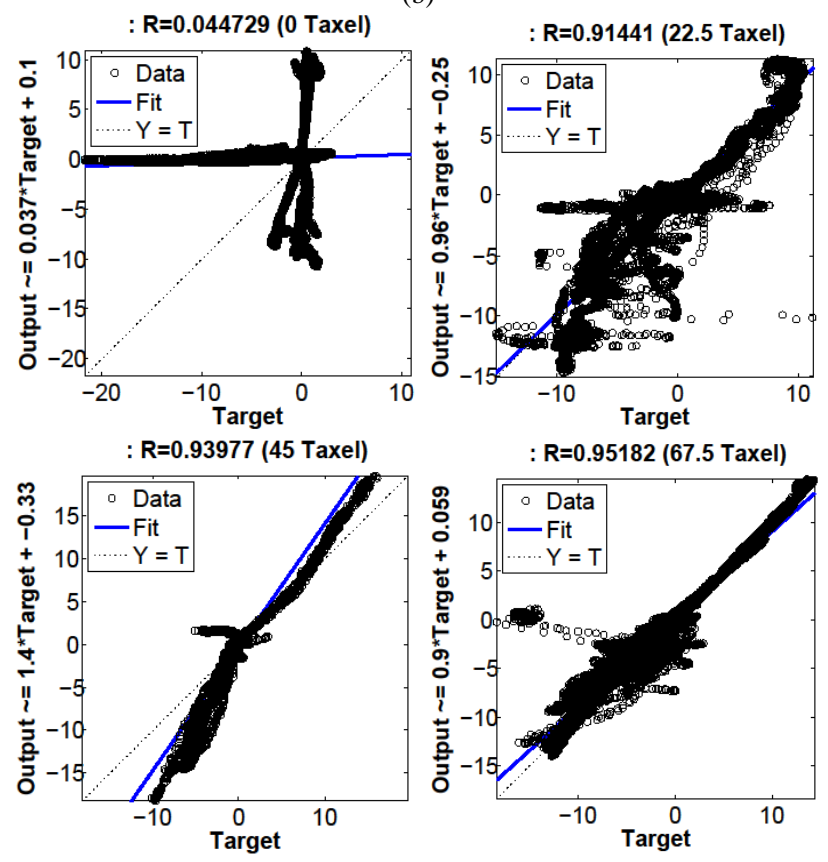

(c)

Figure 9. Results of testing Neural Network with unseen data, X shear direction only. Format is the same as figure 10.

magnitude of the shear force than the planar taxel. The regression plots in Fig. 9(c) continue to show a distinct difference in $\mathrm{R}$ values between the planar and angled taxels. The $45^{\circ}$ taxel again achieves the largest $R$ value. These experimental results show the angled taxels have less error and higher $\mathrm{R}$ values than the planar taxel when measuring forces along the $\mathrm{X}$-Axis.

\section{Forces in $Y$-Axis}

The $0^{\circ}$ planar and $45^{\circ}$ angled taxel display very different behavior when identifying forces along the Y-Axis. The

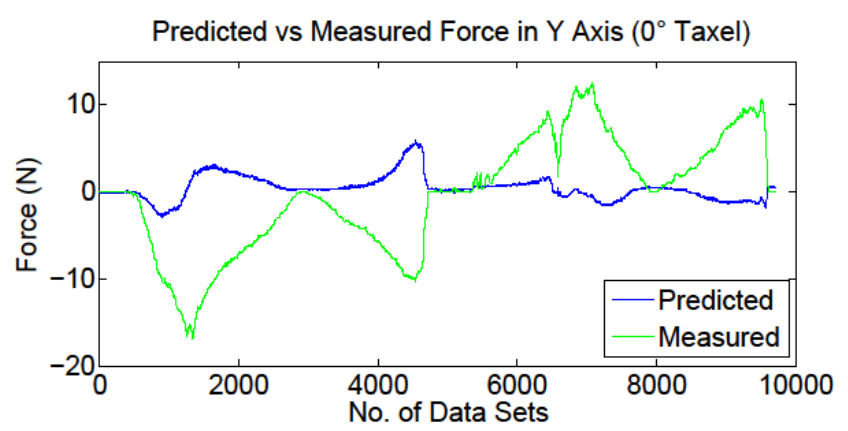

(a)

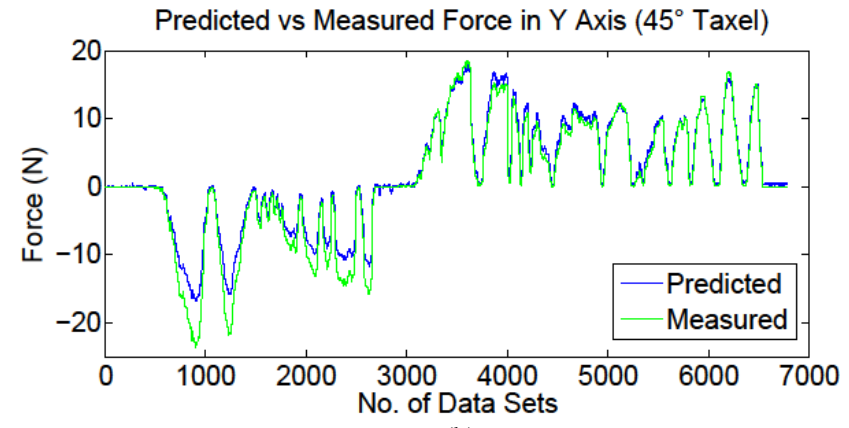

(b)

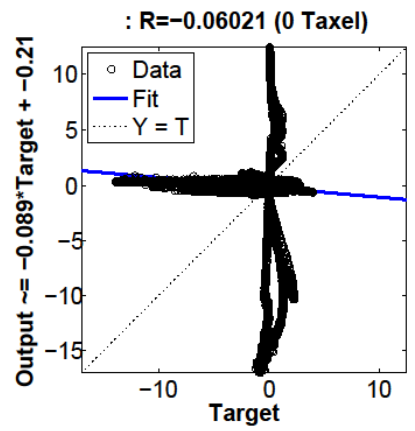

: R=0.96078 (22.5 Taxel)
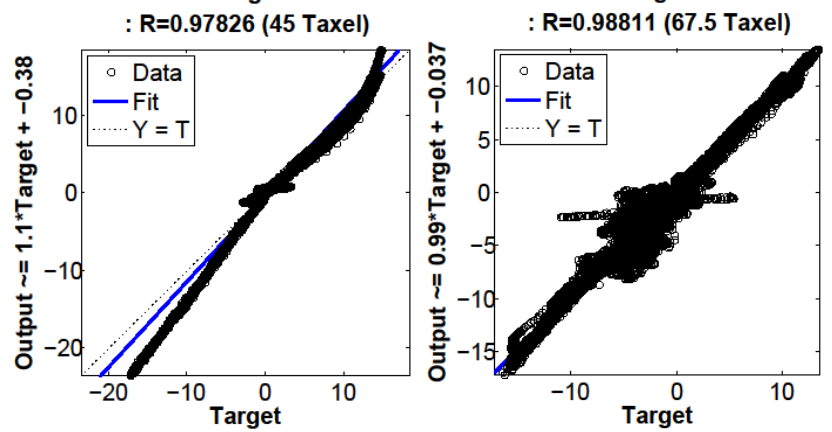

(c)

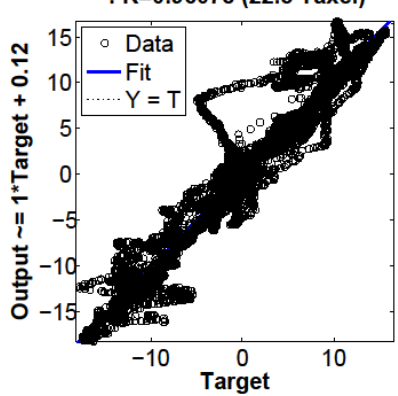

Figure 10. Results of testing Neural Network with unseen data, Y shear direction only. Format is the same as figure 10 and 11.

regression plot of the planar taxel shows a $\mathrm{Y}$-Axis $\mathrm{R}$ value which is smaller than the $\mathrm{X}$-Axis $\mathrm{R}$ value from the previous section. The planar taxel, as seen in Fig. 10(a), no longer correctly determines the direction of the shear force as it did previously. The regression plot of the $45^{\circ}$ taxel shows a YAxis $\mathrm{R}$ value which is slightly larger than the $\mathrm{X}$-Axis $\mathrm{R}$ value from the previous section. This is reflected in Fig. 10(b). The regression plots in Fig. 10(c) follow the trend in $R$ values between the planar and angled taxels. The angled taxels better predict forces along the Y-Axis. 


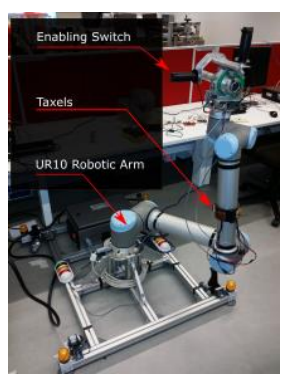

Figure 11. Annotated image of UR10 with taxels mounted.

\section{DEMONSTRATION AND FUTURE WORK}

The primary role of the angled sensor arrays developed in this paper is for pHRI. A preliminary test was conducted to determine the efficacy of angled taxels for control of a robotic arm. The operator's palm was used to apply normal and shear forces to each of the four taxels mounted to a UR10 (Fig. 11). The sensor readings were processed in real time by the neural networks trained in section IV to produce the predicted forces on each taxel. The forces were then combined into a resultant force to unify the multiple points of contact. The resultant force is used to calculate joint velocities in the UR10 controller. Preliminary testing showed the robot movement matching the direction of the applied forces. Further experimentation is needed to quantitatively determine the efficacy of using taxels to control robotic devices.

The future direction for this work is to develop a flexible tri-axial force sensing skin for pHRI. This will involve miniaturizing the taxel unit, making each unit a stand-alone module capable of connecting to other units and increasing the flexibility of the taxel unit. Shrinking the size of a taxel may be possible by reducing the size of the PCB under the pressure sensor. Increasing the flexibility of a taxel unit allows for greater movement when many units are connected into a sensor skin. Possible solutions for this lay in flexible PCBs and 3D printed rubber materials for the sensor base. Small taxels cast in a few millimeters of rubber could be a solution for real freedom of control in pHRI.

\section{CONCLUSION}

This paper presents a new configuration for single axis sensor arrays. The angled sensor configuration was tested against a planar configuration that utilizes the edge effect deformation. The performance of each angled taxel was seen to be better than the planar taxel. The angled taxels were demonstrated practically by using the sensor readings to control a UR10 robot. This result gives merit to the use of angled sensor configurations for the measurement of tri-axial forces.

\section{ACKNOWLEDGMENT}

This work is supported by the Australian Research Council (ARC) Linkage Project (LP140100950), Burwell Technologies, and the Centre for Autonomous Systems (CAS) at the University of Technology Sydney.
The authors would like to acknowledge all members of the JEXO and ANBOT teams for their time and ideas spent on this project.

\section{REFERENCES}

[1] J. T. C. Tan, F. Duan, Y. Zhang, R. Kato, and T. Arai, "Safety design and development of human-robot collaboration in cellular manufacturing," IEEE Int. Conf. Autom. Sci. Eng., pp. 537-542, 2009.

[2] X. Lamy, F. Colledani, F. Geffard, Y. Measson, and G. Morel, "Robotic skin structure and performances for industrial robot comanipulation," IEEE/ASME Int. Conf. Adv. Intell. Mechatronics, pp. 427-432, 2009.

[3] A. Y. Lee and D. Kim, "Detachable tactile sensor skin module for robotic applications," Int. Conf. Ubiquitous Robot. Ambient Intell., pp. 287-288, 2013

[4] Y. Ohmura, Y. Kuniyoshi, and A. Nagakubo, "Conformable and scalable tactile sensor skin for a curved surfaces," IEEE Int. Conf. Robot. Autom., vol. 2006, no. May, pp. 1348-1353, 2006.

[5] J. H. Shan, T. Mei, L. Sun, D. Y. Kong, Z. Y. Zhang, L. Ni, M. Meng, and J. R. Chu, "The design and fabrication of a flexible threedimensional force sensor skin," IEEE/RSJ Int. Conf. Intell. Robot. Syst., no. 60275027, pp. 1965-1970, 2005.

[6] P. Mittendorfer and G. Cheng, "From a multi-modal intelligent cell to a self-organizing robotic skin."

[7] P. Mittendorfer, E. Yoshida, and G. Cheng, "Realizing whole-body tactile interactions with a self-organizing, multi-modal artificial skin on a humanoid robot," Adv. Robot., vol. 29, no. 1, pp. 51-67, 2015.

[8] L. Wang and D. J. Beebe, "Characterization of a silicon-based shearforce sensor on human subjects," IEEE Trans. Biomed. Eng., vol. 49, no. 11, pp. 1340-1347, 2002.

[9] H. Lee, J. Chung, S. Chang, and E. Yoon, "Normal and Shear Force Measurement Using a Flexible Polymer Tactile Sensor With Embedded Multiple Capacitors," J. Microelectromechanical Syst., vol. 17, no. 4, pp. 934-942, 2008

[10] T. Y. Chen, Y. C. Wang, C. Y. Lo, and R. Chen, "Friction-assisted pulling force detection mechanism for tactile sensors," $J$. Microelectromechanical Syst., vol. 23, no. 2, pp. 471-481, 2014.

[11] P. Peng and R. Rajamani, "Flexible microtactile sensor for normal and shear elasticity measurements," IEEE Trans. Ind. Electron., vol. 59, no. 12, pp. 4907-4913, 2012.

[12] T. Chen and Y. Wang, "A novel integrated transparent flexible tactile sensor," Solid-State Sensors, Actuators and Microsystems Conf., pp. 1052-1055, 2011

[13] G. De Maria, P. Falco, C. Natale, and S. Pirozzi, "Integrated Force / Tactile Sensing: The Enabling Technology for Slipping Detection and Avoidance," IEEE Int. Conf. Robot. Autom., pp. 3883-3889, 2015.

[14] G. De Maria, C. Natale, and S. Pirozzi, "Force/tactile sensor for robotic applications," Sensors Actuators, A Phys., vol. 175, pp. 60-72, 2012.

[15] Á. Tar and G. Cserey, "Development of a low cost 3D optical compliant tactile force sensor," IEEE/ASME Int. Conf. Adv. Intell. Mechatronics, pp. 236-240, 2011.

[16] X. Shi, C.-H. Cheng, C. Chao, L. Wang, and Y. Zheng, "A piezoresistive normal and shear force sensor using liquid metal alloy as gauge material," IEEE Int. Conf. Nano/Micro Eng. Mol. Syst., pp. 483-486, 2012.

[17] P. Roberts, D. D. Damian, W. Shan, T. Lu, and C. Majidi, "Softmatter capacitive sensor for measuring shear and pressure deformation," IEEE Int. Conf. Robot. Autom., pp. 3529-3534, 2013.

[18] J. I. Lee, M. G. Kim, M. Shikida, and K. Sato, "A table-shaped tactile sensor for detecting triaxial force on the basis of strain distribution," Sensors (Switzerland), vol. 13, pp. 16347-16359, 2013.

[19] Y. Tenzer, L. P. Jentoft, and R. D. Howe, "The Feel of MEMS Barometers: Inexpensive and Easily Customized Tactile Array Sensors," IEEE Robot. Autom. Mag., no. c, 2014.

[20] L. P. Jentoft, Y. Tenzer, D. Vogt, R. J. Wood, and R. D. Howe, "Flexible, stretchable tactile arrays from MEMS barometers," Int. Conf. Adv. Robot., pp. 1-6, 2013.

[21] M. Y. Chuah and S. Kim, "Enabling Force Sensing During Ground Locomotion: A Bio-Inspired, Multi-Axis, Composite Force Sensor Using Discrete Pressure Mapping," IEEE Sens. J., vol. 14, no. 5, pp. $1693-1703,2014$ 